\title{
Research on Performance of Divesting Firms: Evidence from Listed Corporate in China
}

\author{
Chunxiao Sun, Chunyan Li \\ College of Zhijiang, University of Science and Technology, Hangzhou, Zhejiang, China
}

\begin{abstract}
This study tests the performance of divestiture with the sample of divesting firms listed in the China SSE and SZSE over the time period 2004-08 with a dataset from SINOFIN and RESSET and the methods of factor analysis and independent sample T test. The findings reveal that the divest does not improve the performance in the first and second year after divested, but the impact of divest on firm long-term performance is significant in the third year after divested. The divest improves the performance in the fourth year after divested, but the impact of divestment on firm performance is not significant..
\end{abstract}

Keywords - listed corporate, assets divestiture, performance of divestiture

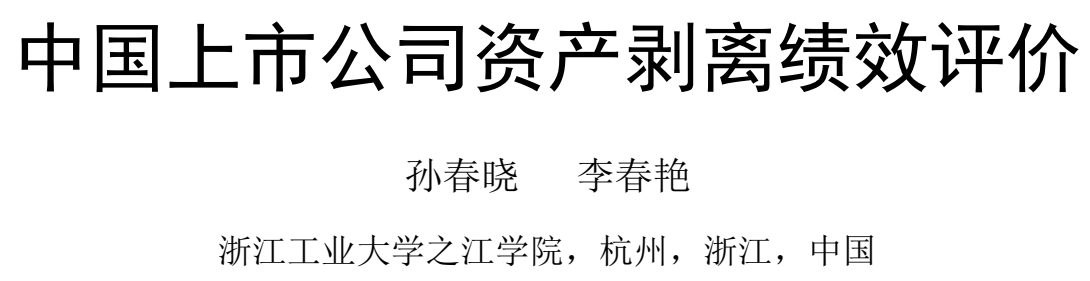

摘 要 本研究选取了在 2004-2008 年期间的在中国沪深证券交易所上市的公司作为样本, 并在 SINOFIN 和 RESSET 研究数据 库收集了样本公司的 2003-2012 年的剥离发生及其相关的财务数据, 应用因子分析法, 结合独立样本 $\mathrm{T}$ 检验, 比较分析了其剥离事件 发生前一年、发生当年和发生后四年上市公司综合绩效得分因子的变化以评价其剥离前后短期和长期绩效的变化。

关键词上市公司, 资产剥离, 剥离绩效

1. 引言

剥离绩效是指剥离发生为股东带来的财富和为公司创 造的价值, 包括财务和市场绩效 (Capron\&Mitchell, 2001), 市场绩效是剥离公告后的市场反应, 表现为短期内累积异 常收益的变化。而已有研究普遍采用财务指标评价剥离的 财务绩效 (Bergh\&Lim, 2008), 选择净资产收益率、总资 产报酬率、每股收益等单一利润率指标。但已有的评价往 往使得剥离绩效的评价过于片面化, 也使评价无法消除操 纵利润的影响 (Hanson\&Song, 2003)。因此, 为全面反映 中国上市公司的剥离业绩, 本研究拟采用综合指标评价法, 采用因子分析法构建综合评价指标体系评价上市公司剥离 绩效, 通过对同一样本公司剥离发生前后的综合因子得分 差值进行独立样本 $\mathrm{T}$ 检验的方法, 分析其剥离前一年、剥

教育部人文社科青年基金项目资助（项目编号：13YJC630139） 浙江工业大学跨国投资研究中心和浙江工业大学之江学院产业经 济学优势学科资助
离当年和剥离后四年综合业绩的变化是否存在显著差异, 对上市公司剥离绩效进行评价。

\section{2. 方法}

\section{1 样本选择和数据来源}

本研究选择 2004-2008 年沪深两市发生剥离交易的上 市公司为样本, 选取了当年仅发生剥离交易, 且发生交易 额大于一千万元人民币，剔除金融类、ST 和 PT 类 的 258 个样本公司, 同时从来自北大经济研究中心的 SINOFIN 数 据库和 RESSET 金融研究数据库, 对应收集其剥离交易和 剥离绩效的数据, 这些窗口数据与上市公司年报数据进行 一定的比对, 实证研究所用软件是 SPSS18.0。

\section{2 变量测量}

本研究选取能反映中国上市公司的获利、偿债、运营、 成长能力的九个财务指标作为因子分析和样本检验的起点 
（夏怡凡, 2010), 包括营业收入增长率、每股收益、净资 产收益率、资产负债率、流动比率和速动比率等。初始变 量定义如表 1 所列。

表 1 初始变量名称及其定义

\begin{tabular}{|l|l|}
\hline 变量名称 & 变量定义 \\
\hline 每股收益 & 税后利润/股本总数 \\
净资产收益率 & 税后净利润 / 平均净资产 \\
流动比率 & 流动资产 / 流动负债 \\
速动比率 & (流动资产一存货 $) /$ 流动负债 \\
资产负债率 & 期末负债总额 / 期末资产总额 \\
营业收入增长率 & 本期主营业务收入/上期主营业务 \\
& 收入-1 \\
每股收益增长率 & 本期每股收益/上期每股收益 -1 \\
净资产增长率 & 本期净资产 $/$ 上期净资产 -1 \\
总资产周转率 & 销售收入净额 / 平均资产总额 \\
\hline
\end{tabular}

\section{3 数据分析}

(1) $\mathrm{KMO}$ 样本测度与 Bartlett 检验

对剥离绩效各测量指标进行因子分析前须进行 KMO 样本测度和 Bartlett 球形检验 (马庆国, 2002)。本研究对 各测量数据是否适合进行因子分析的评判标准, 采用 $\mathrm{KMO}$ 值在 0.90 以上, 非常适合; 0.80-0.90 之间, 很适合; 0.70-0.80 之间, 适合; 0.70 以下, 则不适合 (马庆国, 2002)。并 且要求 Bartlett 统计值的显著性概率小于或等于某一特定 系数 $a$ (通常取 $a=0.01$ ) 时, 可适合进行因子分析。本研 究利用 SPSS18.0 软件对 2004-2008 年财务数据进行 KMO 和 Bartlett 检验结果表明其显著性概率值均为 0.000 , 因此, 在 0.01 的显著水平下通过检验, 应拒绝变量相互独立的假 设, 即各测量变量间具有较强相关性。且 2004-2008 各年 的 KMO 统计量均在 0.7 以上, 适合作因子分析, 说明变量 选取较科学, 分析结果较理想。

\section{（2）总方差的解释}

通过因子分析可得到各测量变量总方差解释。根据因 子个数提取原则的两个标准：因子对应的特征值大于 1 的 前 $\mathrm{m}$ 个主成分和前 $\mathrm{m}$ 个因子累计贡献率大于 $85 \%$ 。应用主 成分法并选用方差最大法对上市公司绩效全部测量指标数 据进行探索性因子分析, 结果得到三个特征值大于 1 的因 子, 2004-2008 各年前三个初始因子特征值大于 1, 且累计 方差贡献率分别为 $77.60 \% 、 76.26 \% 、 74.47 \% 、 65.88 \%$ 、 $71.78 \%$ 。除 07 年方差累计贡献率小于 $70 \%$ 之外, 其余前三 个初始因子方差累计贡献率均达到 $75 \%$ 左右, 说明前三个 因子足以描述上市公司的综合绩效水平。
（3）旋转后的因子载荷矩阵说明

通过旋转初始因子载荷矩阵, 可使因子载荷矩阵中系 数更显著, 重新分配因子和原始变量间关系, 各因子在各 项指标上载荷更明确, 从而解释性增强。利用方差极大法 进行旋转后, 得到 2004-2008 年的旋转后的因子载荷阵和 描述性统计结果。根据统计结果表明, 因子 F1 在每股收益、 净资产收益率、每股收益增长率和净资产增长率上载荷较 大, 因此 F1 为反映盈利能力的综合指标; 因子 F2 在资产 负债率、速动比率以及流动比率上载荷较大, 因此 $\mathrm{F} 2$ 为反 映公司偿债能力的综合指标; 因子 F3 在营业收入增长率、 总资产资产周转率上的载荷较大, 均大于 0.5 , 为反映资产 管理能力的综合指标。

(4) 不同年度综合因子的得分函数

通过计算各因子对应方差贡献率与因子累计方差贡献 率总和的比值为权重, 本研究得到 2004-2008 年度各综合 因子得分的函数:

2004 年剥离综合绩效因子的得分函数:

$$
\mathrm{F}_{04}=0.5376 \mathrm{~F}_{04-1}+0.3052 \mathrm{~F}_{04-2}+0.1572 \mathrm{~F}_{04-3}
$$

2005 年剥离综合绩效因子的得分函数:

$$
\mathrm{F}_{05}=0.4863 \mathrm{~F}_{05-1}+0.3518 \mathrm{~F}_{05-2}+0.1619 \mathrm{~F}_{05-3}
$$

2006 年剥离综合绩效因子的得分函数:

$$
\mathrm{F}_{06}=0.4318 \mathrm{~F}_{06-1}+0.3707 \mathrm{~F}_{06-2}+0.1975 \mathrm{~F}_{06-3}
$$

2007 年剥离综合绩效因子的得分函数:

$$
\mathrm{F}_{07}=0.3788 \mathrm{~F}_{07-1}+0.3274 \mathrm{~F}_{07-2}+0.2938 \mathrm{~F}_{07-3}
$$

2008 年剥离综合绩效因子的得分函数:

$$
\mathrm{F}_{08}=0.4202 \mathrm{~F}_{08-1}+0.3927 \mathrm{~F}_{08-2}+0.1871 \mathrm{~F}_{08-3}
$$

\section{（5）剥离综合绩效得分}

通过将样本上市公司 2004-2008 年各单项因子得分 $\left(F_{i t-1} 、 F_{i t-2} 、 F_{i t-3}\right)$ 代入综合因子得分函数 $F_{0 i}$, 得到综合 得分因子 $\mathrm{F}$ 。因此, 可根据综合因子得分函数计算得到的 2004-2008 年度发生剥离样本公司的综合因子得分值及其 描述性统计值, 如表 2 所示。并以此计算样本公司剥离发 生前一年、剥离发生当年以及剥离发生后四年的的上市公 司综合的短期和长期绩效得分及其结果的描述性统计, 如 表 3 所列。

\section{3. 结论}

基于上述分析得到了上市公司剥离发生前一年、当年、 后四年的综合绩效因子得分后, 本研究进一步计算其因子 
得分的差额。基于样本量足够大, 按中心极限定理, 可从 大样本的近似分布的角度来分析问题, 每个总体的随机均 值函数都可认为近似于服从了正态分布, 符合 $\mathrm{T}$ 检验的基 本要求, 可将通过独立样本 $\mathrm{T}$ 检验, 对样本公司发生剥离 前一年、当年和后四年的综合因子得分差进行检验, 比较 其是否存在显著差异, 对上市样本公司剥离短期和长期综 合绩效做出初步评价。实现对上市样本公司剥离前后剥离 综合绩效因子得分差值的检验, 独立样本 $\mathrm{T}$ 检验的双尾检 验结果如表 4 所列。

表 2 2004-2008 年上市公司剥离绩效综合因子得分描述性统计

\begin{tabular}{|l|llllll|}
\hline 变量 & 统计量 & 2004 & 2005 & 2006 & 2007 & 2008 \\
\hline F1 & 极小值 & -1.649 & -4.113 & -2.577 & -3.901 & -1.615 \\
& F2 极大值 & 5.472 & 1.169 & 3.111 & 2.860 & 4.255 \\
& 均值 & 0.243 & -0.074 & 0.286 & -0.189 & 0.155 \\
F3 & 极小值 & -4.507 & -1.185 & -4.768 & -2.259 & -3.066 \\
& 极大值 & 2.223 & 5.109 & 1.881 & 5.311 & 4.165 \\
& 均值 & 0.201 & 0.003 & -0.222 & 0.098 & -0.111 \\
& 极小值 & -0.977 & -3.678 & -1.227 & -1.986 & -1.819 \\
& 极大值 & 5.559 & 2.286 & 2.797 & 5.875 & 4.251 \\
& 均值 & 0.157 & 0.029 & 0.157 & 0.181 & 0.083 \\
& 极小值 & -1.10 & -2.28 & -1.89 & -2.26 & -1.71 \\
& 极大值 & 3.14 & 1.84 & 1.11 & 1.77 & 1.54 \\
& 均值 & 0.141 & 0.001 & -0.088 & 0.049 & -1.04 \\
& 标准差 & 0.622 & 0.615 & 0.596 & 0.579 & 0.595 \\
& 样本数 & 45 & 50 & 43 & 65 & 55 \\
\hline
\end{tabular}

从剥离前后样本公司综合绩效因子得分的独立样本 $\mathrm{T}$ 检验结果可得出, 公司剥离后第一年的综合因子得分 (F1)、 剥离后第二年的综合因子得分 (F2) 的综合绩效得分均值, 与剥离当年的综合绩效得分 (F0) 均值相比, 不但没有上 升, 反而出现了不同程度的下降。而剥离后第三年的综合 因子得分 (F3) 以及剥离后第四年的综合因子得分 (F4) 与剥离当年的综合绩效得分 (F0) 均值比较, 出现了一定 程度的增长, 但除了 F3-F0 (剥离发生后第三年与剥离当年 的综合绩效因子得分差）的显著性概率值为 0.096 , 在 0.1 的水平下通过了显著性检验外, 其余各项的剥离绩效差值 均未通过显著性检验, 如表 4 所列。说明剥离发生后的第 三年上市公司业绩出现了显著上升, 而且 F4-F-1（剥离后 第四年与剥离发生前一年的综合绩效因子得分差)和 F4-F0
（剥离后第四年与剥离发生当年的综合绩效因子得分差） 均为正值, 一定程度表明上市公司长期的剥离绩效出现上 升, 而且较为显著。

表 3 剥离前一年和剥离后四年的综合因子得分描述性统计

\begin{tabular}{|c|c|c|c|c|c|c|}
\hline 变量 & 统计量 & 前一年 & 第一年 & 第二年 & 第三年 & 第四年 \\
\hline \multirow[t]{3}{*}{$\mathrm{F} 1$} & 极小值 & -1.79 & -1.12 & -4.715 & -1.821 & -3.465 \\
\hline & 极大值 & 1. 64 & 2. 79 & 2. 121 & 5. 168 & 1. 281 \\
\hline & 均值 & 0.149 & -0.082 & 0.056 & -0.069 & -0.195 \\
\hline \multirow[t]{3}{*}{$\mathrm{F} 2$} & 极小值 & -2.607 & -3.985 & -1.578 & -1.252 & -2.566 \\
\hline & 极大值 & 3. 123 & 2. 705 & 4. 155 & 3. 134 & 1.811 \\
\hline & 均值 & 0.561 & -0.063 & 0.253 & 0.148 & 0.013 \\
\hline \multirow[t]{3}{*}{ F3 } & 极小值 & -1.329 & -1.835 & -3.991 & -1.986 & -2.736 \\
\hline & 极大值 & 2. 453 & 1.135 & 4. 276 & 5.875 & 3.915 \\
\hline & 均值 & 0.105 & -0.087 & -0.126 & 0.181 & 0.232 \\
\hline \multirow[t]{3}{*}{$\mathrm{F}$} & 极小值 & -2.350 & -3.287 & -3.597 & -5.361 & -2.913 \\
\hline & 极大值 & 5. 121 & 4. 295 & 2. 651 & 2.725 & 1.747 \\
\hline & 均值 & 0.121 & -0.061 & 0.058 & 0.384 & -0.046 \\
\hline
\end{tabular}

比较样本公司剥离当年和剥离后四年综合绩效的因子 得分 F1、F2、F3、F4 与剥离前一年综合得分 F-1 的差值, 得到 F0-F-1 可以看出, F0-F-1 的检验值为 0.037 且通过了 0.05 水平下的显著性检验, 说明剥离的短期绩效是有所改 善的。 F3-F-1 的检验值为 0.053 且通过了 0.1 水平下的显 著性检验, 这说明剥离后第三年与剥离前一年相比, 公司 的业绩得到了一定程度的提升。F1-F-1、F2-F-1 均为负值, F4-F-1 的均值为正, 但未通过显著性检验。因此, 以剥离 前一年上市公司的综合绩效为基数, 剥离后第一年、第二 年的业绩反而出现下降, 而剥离后第三年的业绩出现较为 显著的上升, 剥离后第四年的业绩仍有上升, 但并不显著。 该研究结果也可说明一定程度上中国上市公司剥离交易发 生后长期的剥离绩效出现上升, 而且剥离绩效改善的较为 显著。

基于上述分析表明，资产剥离有利于显著改善公司剥 离发生当年的综合绩效, 而且剥离交易发生后第三年和第 四年的绩效也得到了较为显著的改善。因此, 后续的研究 将可以以 F0-F-1（剥离发生的当年与剥离前一年的综合绩 效因子得分差）代表剥离的短期综合绩效、F3-F0（剥离发 生后第三年与剥离当年综合绩效因子得分差) 和 F3-F-1 (剥 离发生后第三年与剥离前一年综合绩效因子得分差）表示 剥离后的长期综合绩效, 通过回归分析等方法探寻影响剥 离发生的样本公司绩效的因素, 以积极地提升剥离绩效。 
表 4 剥离前后综合绩效因子得分差的独立样本 T 检验结果

\begin{tabular}{|c|c|c|c|}
\hline 对比检验年度 & 均值 & 正值比率 $(\%)$ & 显著性 \\
\hline 剥离当年与前一年 $\left(\mathrm{F}_{0}-\mathrm{F}_{-1}\right)$ & 0.0163 & 47.67 & $0.037^{* *}$ \\
\hline 剥离后一年与当年 $\left(\mathrm{F}_{1}-\mathrm{F}_{0}\right)$ & -0.0293 & 52.71 & 0.895 \\
\hline 剥离后二年与当年 $\left(\mathrm{F}_{2}-\mathrm{F}_{0}\right)$ & -0.019 & 49.26 & 0.696 \\
\hline 剥离后三年与当年 $\left(\mathrm{F}_{3}-\mathrm{F}_{0}\right)$ & 0.3268 & 52.52 & $0.096^{*}$ \\
\hline 剥离后四年与当年 $\left(\mathrm{F}_{4}-\mathrm{F}_{0}\right)$ & 0.0487 & 44.21 & 0.397 \\
\hline $\begin{array}{c}\text { 剥离后一年与剥离前一年 } \\
\left(\mathrm{F}_{1}-\mathrm{F}_{-1}\right)\end{array}$ & -0.013 & 46.51 & 0.997 \\
\hline $\begin{array}{c}\text { 剥离后二年与剥离前一年 } \\
\left(\mathrm{F}_{2}-\mathrm{F}_{-1}\right)\end{array}$ & -0.0243 & 41.87 & 0.995 \\
\hline $\begin{array}{c}\text { 剥离后三年与剥离前一年 } \\
\left(\mathrm{F}_{3}-\mathrm{F}_{-1}\right)\end{array}$ & 0.1362 & 42.75 & $0.053^{*}$ \\
\hline $\begin{array}{c}\text { 剥离后四年与剥离前一年 } \\
\left(\mathrm{F}_{4}-\mathrm{F}_{-1}\right)\end{array}$ & 0.0527 & 48.95 & 0.236 \\
\hline
\end{tabular}

附注1: $\mathrm{F}_{-1} 、 \mathrm{~F}_{0} 、 \mathrm{~F}_{1} 、 \mathrm{~F}_{2} 、 \mathrm{~F}_{3} 、 \mathrm{~F}_{4}$ 分别表示剥离前一年、当年、剥离后 第一年、第二年、第三年、第四年公司的综合绩效因子得分。

*、**分别代表双侧检验在 $10 \%$ 、5 \%的水平上显著。

\section{参考文献(References)}

[1] L. Capron, W. Mitchell, \& A. Swaminathan, "Asset divestiture following horizontal acquisitions: a dynamic view," Strategic Management Journal, 2001, 22 (9), 817-844.

[2] D.-D. Bergh, \& E.-N.-K. Lim, "Learning how to restructure: absorptive capacity and improvisational views of restructure actions and performance," Strategic Management Journal, 2008(29), 593-616.

[3] R. C. Hanson, M. H. Song, "Long term performance of divesting firms and the effect of managerial ownership." Journal of Economics and Finance, 2003, 27 (3), 321-336.

[4] Q.-G. Ma, "Management Statistics," Beijing: Science Press, 2002, pp.320 